\title{
PNA-NLS conjugates as single-molecular activators of target sites in double-stranded DNA for site-selective scission
}

\author{
Yuichiro Aiba, ${ }^{\S * a, b}$ Yuya Hamano, ${ }^{b}$ Wataru Kameshima, ${ }^{b}$ Yasuyuki Araki, ${ }^{c}$ Takehiko Wada, ${ }^{c}$ Alessandro \\ Accetta, ${ }^{\S d}$ Stefano Sforza, ${ }^{\S d}$ Roberto Corradini, ${ }^{\S * d}$ Rosangela Marchelli $^{\S d}$ and Makoto Komiyama ${ }^{* a, b}$
}

\author{
${ }_{5}$ Received (in $\left.X X X, X X X\right)$ Xth $X X X X X X X X X 20 X X$, Accepted Xth XXXXXXXXX 20XX \\ DOI: $10.1039 / b 000000 x$
}

\begin{abstract}
Artificial DNA cutters have been developed by us in previous works by combining two strands of pseudo-complementary peptide nucleic acid (pcPNA) with Ce(IV)-EDTA-promoted hydrolysis. The pcPNAs have two modified nucleobases (2,6-diaminopurine and 2-thiouracil) instead of conventional A 10 and T, and can invade double-stranded DNA to activate the target site for the scission. This system has been applied to site-selective scissions of plasmid, $\lambda$-phage, $E$. coli genomic DNA, and human genomic DNA. Here, we reported a still simpler and more convenient DNA cutter obtained by conjugating peptide nucleic acid (PNA) with a nuclear localization signal (NLS) peptide. This new DNA cutter requires only one PNA strand (instead of two) bearing conventional (non-pseudo-complementary) nucleobases. This

15 PNA-NLS conjugate effectively activated the target site in double-stranded DNA and induced siteselective scission by Ce(IV)-EDTA. The complex formation between the conjugate and DNA was concretely evidenced by spectroscopic results based on time-resolved fluorescence. The target scission site of this new system was straightforwardly determined by the Watson-Crick rule, and mismatched sequences were clearly discriminated. Importantly, even highly GC-rich regions, which are difficult to be 20 targeted by previous strategy using pcPNA, were successfully targeted. All these features of the present DNA cutter are promising for various future applications.
\end{abstract}

\section{Introduction}

Naturally occurring restriction enzymes have too poor sitespecificity to manipulate huge DNAs such as human genome, and 25 thus new tools are required for further developments of molecular biology and biotechnology. Various attempts have been already made to cut DNA site-selectively. ${ }^{1-5}$ Recently, a chemistry-based artificial restriction DNA cutter (ARCUT) ${ }^{5,6}$ for site-selective hydrolysis of double-stranded DNA was prepared by combining ${ }_{30} \mathrm{Ce}(\mathrm{IV})$-EDTA complex as molecular scissors ${ }^{7}$ and two strands of pseudo-complementary peptide nucleic acid (pcPNA) ${ }^{8-11}$ (Fig. 1a (i)). Two pcPNA strands invade double-stranded DNA at target site ("double-duplex invasion") ${ }^{10}$ and form single-stranded portions as hot spots which are preferentially hydrolyzed by ${ }_{35} \mathrm{Ce}$ (IV)-EDTA due to its intrinsic substrate specificity (Ce(IV)EDTA preferentially hydrolyzes single-stranded DNAs much faster than double-stranded DNAs ${ }^{7}$ ). In our previous report, even human genome, whose length is about three billion bases, was cut at the target site ${ }^{12,13}$ ). In order to form the invasion complex and 40 activate the target site efficiently, two strands of pcPNA, in which conventional nucleobases $\mathrm{A}$ and $\mathrm{T}$ are replaced by 2,6diaminopurine and 2-thiouracil, must be used. With this strategy, however, GC-rich regions are difficult to be targeted, since pseudo-complementary bases for $\mathrm{G}$ and $\mathrm{C}$, which strongly bind to 45 natural bases $\mathrm{C}$ and $\mathrm{G}$ but hardly form a mutual base-pair, have not yet been fully developed. ${ }^{14-16}$ Accordingly, still simpler and more convenient DNA cutters should be obtainable if [I] the number of PNA strands to activate a predetermined site in double-stranded DNA is reduced from two to one and [II] 50 conventional PNAs involving no pseudo-complementary bases can be used in place of pcPNAs.

Several reports have indicated the possibility of performing dsDNA invasion using single PNA strand. Corey's group reported that invasion activity of PNA is enhanced when 55 positively-charged amino acids or oligopeptides are attached to the PNA, in case that target site had a special sequence (e.g., inverted repeats). ${ }^{17}$ Backbone- or nucleobase-modified PNA was more recently shown by Ly's group to perform strand invasion. ${ }^{18,}$ ${ }^{19}$ When attached to the nuclear localization signal (NLS) peptide 60 of SV40 (PKKKRKV), conventional PNA (without either backbone- or nucleobase-modification) showed anti-gene effects consistent with a strand invasion process. ${ }^{20-25}$ We recently used a PNA-NLS conjugate to selectively target the N-myc oncogene $(M Y C N)$ in neuroblastoma, showing evident anti-gene effect. ${ }^{23}$

${ }_{65}$ This strategy was also successful in inhibiting growth of other tumor types such as rhabdomyosarcoma in vivo, ${ }^{24}$ and spectroscopic data suggested that the NLS-conjugated PNA interacts with the short DNA involving its target sequence, ${ }^{25}$ and this process operates also in cellular systems, at least during DNA 70 transcription. In this paper, we use PNA-NLS conjugates as single-molecular activators of predetermined sites in double- 


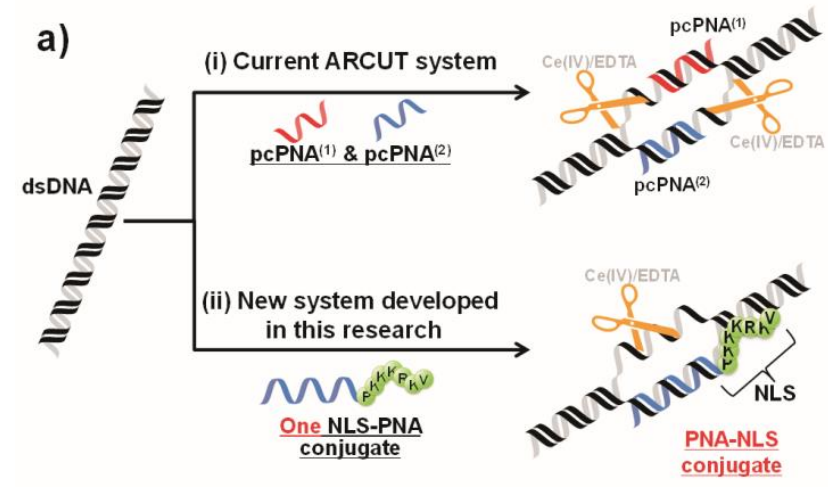

b)

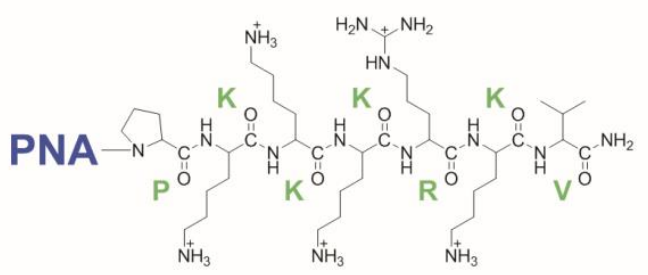

Fig. 1 a) Schematic representations of (i) site-selective DNA cutter using two pcPNA strands (ARCUT) and (ii) the present DNA cutter using only one strand of NLS-attached conventional PNA. b) Chemical structure of 5 PNA-NLS conjugate. The NLS was directly attached to the C-terminus of conventional PNA bearing no pseudo-complementary bases.

stranded DNA. The hot spot, formed by one strand of the conjugate at target site in the DNA, is selectively hydrolyzed by Ce(IV)-EDTA (Fig. 1a (ii)). Targeted scission sequences can be 10 almost freely chosen, and are never restricted to inverted repeats as previously reported. ${ }^{17}$ Even the sequences composed of only GC base-pairs, which are hard to be targeted by double-duplex invasion of pcPNAs, can be cut site-selectively by this new system. Both of the two requirements [I] and [II] for widely 15 applicable DNA cutters, presented above, are satisfactorily fulfilled.

\section{Results and discussion}

Site-selective DNA scission by combining one strand of PNANLS conjugate and Ce(IV)-EDTA

20 As shown in Fig. 2a, a sequence (16-bp) in exon 2 of $N$-myc oncogene $(M Y C N)$ was incorporated in a plasmid DNA $(3,563$ bp), and was targeted by a PNA1-NLS/Ce(IV)-EDTA system. The NLS was directly attached to the C-terminus of 16-mer PNA1 (Fig. 1b), which is complementary to the sense strand 25 (T340-A355) of $M Y C N .^{24}$ This target sequence is almost random and contains no inverted repeat. When the PNA1 binds to the sense strand according to the Watson-Crick rule, the corresponding antisense strand becomes single-stranded (see Fig. 1a (ii)) and is preferentially hydrolyzed by Ce(IV)-EDTA (this 30 catalyst hardly hydrolyzes double-stranded $\mathrm{DNA}^{7}$ ). It should be noted that PNA1 involves only conventional nucleobases and no pseudo-complementary bases are used (the requirement [II]). The site-selective hydrolysis of DNA was carried out at $\mathrm{pH} 7.0$ and $37^{\circ} \mathrm{C}$ unless otherwise noted (see Supporting Information for 35 experimental details). As shown in Supplementary Fig. 2, the form I DNA (supercoiled) was first converted to the form II (relaxed open-circular) and then to the form III (linear). In order to pin down the scission site, the reaction mixtures were further digested with a restriction enzyme Sma $\mathrm{I},{ }^{\ddagger}$ and the products were 40 analyzed by $0.8 \%$ agarose gel electrophoresis (see Supplementary Fig. 3a). Only two scission bands were observed in the gel (lane 3 in Fig. 2a), confirming that the scission was site-selective. The sizes of these two bands are exactly identical to those (around 1.5 and $2.0 \mathrm{kbp}$ ) expected from the double 45 digestion by the PNA1-NLS/Ce(IV)-EDTA system and Sma I (site 2,373). To validify the universality of PNA-NLS/Ce(IV)EDTA system, a 16-bp region in blue fluorescent protein (BFP), which is also almost random sequence, was targeted in Fig. 2 b. The scission by the PNA2-NLS/Ce(IV)-EDTA system (followed 50 by digestion with $\mathrm{Xba} \mathrm{I})^{\star}$ provided two fragments corresponding to the scission at the binding site of PNA2 (lane 3). It was concluded that only one strand of PNA-NLS conjugate is sufficient to activate the target site in double-stranded DNA and induce the site-selective scission by Ce(IV)-EDTA (the 55 requirement $[\mathrm{I}])$. In the absence of the PNA-NLS conjugate, Ce(IV)-EDTA never cut the DNA (lanes 2 in Fig. 2a and 2b), and no DNA scission occurred when PNA1 bearing no NLS-peptide was used in place of PNA1-NLS (Supplementary Fig. 4).

In order to shed light on the role of the NLS of SV40 in the 60 conjugates, another positively charged peptide (RRRRR) was instead attached to the C-terminus of PNA2. When this PNA2RRRRR conjugate was combined with Ce(IV)-EDTA for DNA hydrolysis, non-targeted sites were notably cut and the scission at the target site in the BFP gene was never a dominant process 65 (data not presented). Apparently, this non-NLS conjugate binds to the DNA rather randomly through electrostatic interactions and activates various sites in this DNA. Critical importance of the primary structure of the NLS, its tertiary structure and/or hydrophilicity/hydrophobicity balance is strongly indicated. Both 70 the PNA and the NLS portions in the conjugate are crucial for the present site-selective scission of double-stranded DNA. In a proposed mechanism, the NLS in the NLS-PNA conjugates interacts with DNA to induce a change in its local conformation and allows the PNA to invade the site without the requirement of

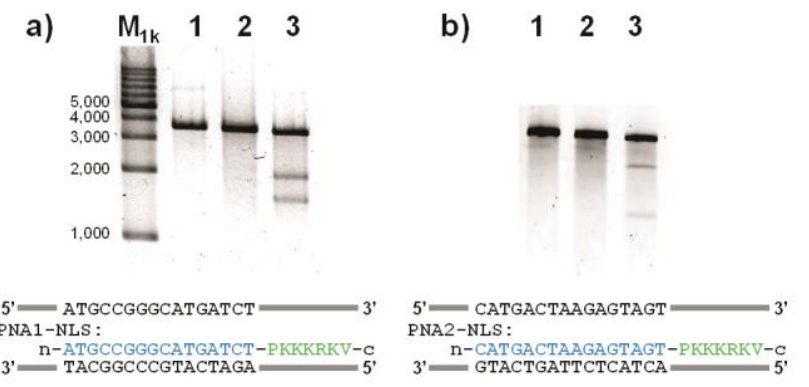

75

Fig. 2 Site-selective DNA scission by combining one strand of PNA-NLS conjugate and Ce(IV)-EDTA. In a) and b), a 16-bp sequence in the exon 2 of MYCN gene and BFP gene in a plasmid were targeted, respectively. Lane 1, DNA only (control); Lane 2, DNA treated with Ce(IV)-EDTA 80 (control); Lane 3, after the scission with the combination of Ce(IV)EDTA and PNA1-NLS conjugate (for a)) or PNA2-NLS (for b). Reaction conditions: $[\mathrm{DNA}]=4.0 \mathrm{nM}$, $[$ PNA1-NLS or PNA2-NLS $]=200 \mathrm{nM}$, $[\mathrm{Ce}(\mathrm{IV})-\mathrm{EDTA}]=200 \mu \mathrm{M},[\mathrm{HEPES}(\mathrm{pH} 7.0)]=5.0 \mathrm{mM}$ and $[\mathrm{NaCl}]=$ $100 \mathrm{mM}$ at $37^{\circ} \mathrm{C}$ for 3 days. The scission mixture was treated with Sma I 85 (for a)) or with Xba I (for b)) before the electrophoresis (stained with GelStar $^{\circledR}$ from FMC). The scission sites of the restriction enzymes are presented in Supplementary Figure 3. 
inverted repeats. The single-stranded portion, formed in the counter-strand, is hydrolyzed by Ce(IV)-EDTA, as schematically depicted in Fig. 1a (ii). This proposal is consistent with previous results that an NLS peptide of analogous sequence 5 (KPKKKKEK) strongly interacts with double-stranded DNA and invokes remarkable changes in its structure. ${ }^{26}$

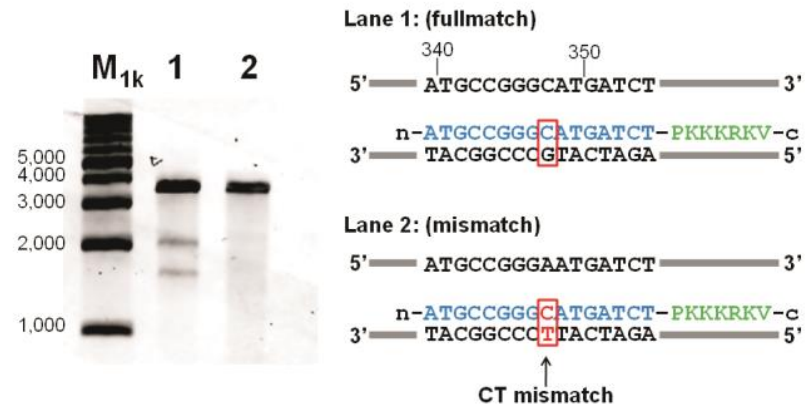

Fig. 3 Mismatch discrimination by PNA1-NLS/Ce(IV)-EDTA system. Lane 1, fully-matched DNA; Lane 2, DNA involving a CT mismatch to 10 PNA1. Reaction conditions: $[$ DNA] $=4.0 \mathrm{nM},[$ PNA1-NLS $]=200 \mathrm{nM}$, $[\mathrm{Ce}(\mathrm{IV})-\mathrm{EDTA}]=200 \mu \mathrm{M},[\mathrm{HEPES}(\mathrm{pH} 7.0)]=5.0 \mathrm{mM}$ and $[\mathrm{NaCl}]=$ $100 \mathrm{mM}$ at $37^{\circ} \mathrm{C}$ for 3 days. After the DNA scission, the reaction mixtures were treated with Sma I before the gel electrophoresis. The scission site of Sma I is presented in Supplementary Figure 3.

\section{Mismatch-discrimination ability of PNA-NLS conjugate}

For the confirmation of the mismatch-discrimination ability of PNA-NLS conjugate, the Ce(IV)-EDTA scission experiment with target DNA bearing one base-pair difference was conducted. In Fig. 3, the CG base-pair in the center of the 16-bp target sequence 20 of PNA1-NLS (at the position 348 in Fig. 3) was changed to an AT pair, and thus one CT mismatch was introduced between the sense strand of MYCN and the conjugate. Even with this subtle change, the DNA scission vanished (compare lane 1 with lane 2 in Fig. 3). Thus, the present DNA-cutting system satisfactorily 25 distinguishes between fully-matched target sequence and slightly different sequences. The electrostatic interactions between the positive charges of the NLS and the DNA do not deteriorate the site-specificity, but simply strengthen the binding between the conjugate and target DNA to the extent required for the strand 30 invasion. Thereafter, the scission site is precisely determined by the Watson-Crick rule and can be freely chosen according to experimental needs.

Confirmation of complex formation between PNA-NLS conjugate and the target DNA by fluorescence spectroscopy

${ }_{35}$ Fluorescent (FL) spectroscopic studies should give us evidences on complex formation and detailed information and insight on these PNA-NLS conjugates - target plasmid DNA complexes. For this purpose, a Quasar 570 dye (from BIOSEARCH TECHNOLOGIES, INC) was covalently bound to the N-terminus 40 of PNA2-NLS conjugate (Fig. 4a). FL spectral change, especially FL intensity increment of Quasar 570 and Cy3 family is qualitatively indicated complex formations between DNA's, in general. ${ }^{27}$ Extremely enhanced FL intensity from the Dye-PNA2NLS conjugate was observed in the presence of the plasmid (the 45 red line), while very weak FL in the absence (the black line in Fig. 4b). FL lifetime studies would give us more quantitative information on complex formation. The decay profiles were also a)
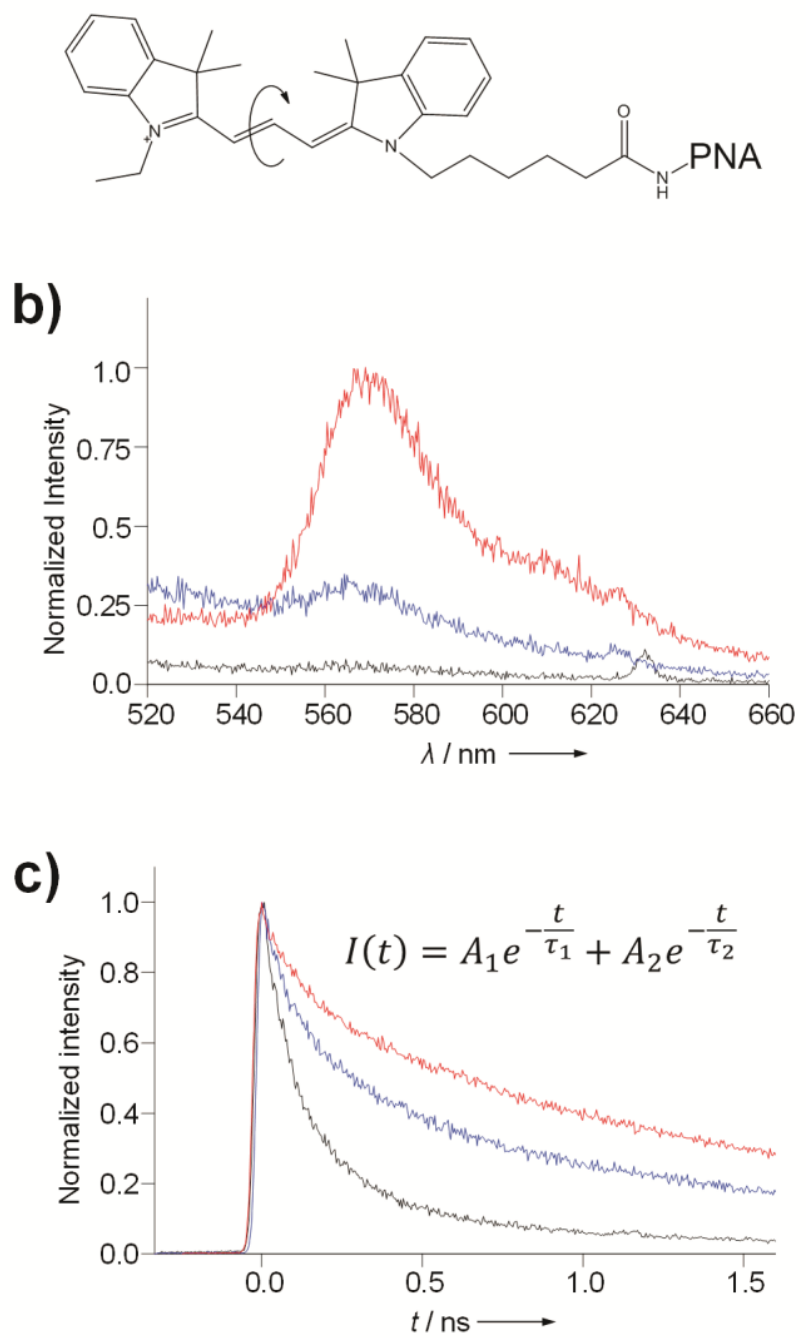

Fig. 4 a) Structure of the conjugate between Quasar 570 dye and PNA250 NLS. b) Normalized fluorescence spectra (excitation: $395 \mathrm{~nm}$, photon counting: from $1.0 \mathrm{~ns}$ to $1.2 \mathrm{~ns}$ after laser irradiation) and c) fluorescence decay curves (excitation: $395 \mathrm{~nm}$, photon counting: from $550 \mathrm{~nm}$ to 620 nm). Black, Dye-PNA2-NLS alone; red, Dye-PNA2-NLS + the plasmid. The values of $\tau 1$ (s), $\tau 2$ (s), A1, and A2, obtained by fitting of the curves 55 in c), are $(0.14,0.91,0.82,0.18)$ and $(0.18,1.72,0.27,0.73)$, respectively. For the purpose of comparison, the results of the addition of the 16-mer complementary oligonucleotide are shown by the blue curves (the fluorescence parameters are listed in Supplementary Table 1, together with the values for the other systems). Measurement conditions are [Dye60 PNA2-NLS] $=500 \mathrm{nM}$, [the DNA] $=0$ or $1000 \mathrm{nM}$, and [phosphate buffer $(\mathrm{pH} 7.0)]=5 \mathrm{mM}$.

remarkably changed by the plasmid addition (Fig. 4c). In all the systems, the decay curves were satisfactorily fit using two exponential terms, indicating that the photo-excited singlet state ${ }_{65}$ of the dye should have at least two states. Without the plasmid, the shorter lifetime term $\left(\mathrm{t}_{1}=0.14 \mathrm{~ns}\right)$ was overwhelmingly the major component $\left(\mathrm{A}_{1}=82 \%\right.$; the black line). With the plasmid, however, the longer lifetime component $\left(t_{2}=1.72 \mathrm{~ns}\right)$ became dominant $\left(\mathrm{A}_{2}=73 \%\right.$; the red line $)$. These results clearly indicate 70 that the addition of the plasmid has induced increment of the long lifetime singlet state component of the dye. According to previous studies on the fluorescence from a similar dye of $\mathrm{Cy} 3$ 
family, the emission efficiency is primarily governed by the photo-induced cis-trans isomerization in the photo-excited singlet state of the dye and quantum yield of photo-isomerization sharply depends upon micro-environmental freedom and interactions with 5 aromatic residues nearby the chromophore. ${ }^{27}$ The complex formed in the present study involves two DNA strands and one PNA strand, so that the Quasar 570 dye, bound to the PNA2-NLS, is placed in a sterically restricted field. Furthermore, there are many adjacent nucleobases which could interact with the dye. On 10 account of these two factors, the photo-isomerization of the dye is notably suppressed, resulting in the increases in both the fluorescence intensity and the lifetime. Therefore, the increment of the long lifetime component with the plasmid addition clearly indicates the complex formation between the PNA2-NLS 15 conjugate and its target plasmid DNA. Consistently, with the use of the complementary oligonucleotide in place of the plasmid, similar increases in both fluorescence parameters were observed but to a much smaller extent (the blue curves in Fig. $4 \mathrm{~b}$ and c). The NLS sequence in the conjugate is known to be recognized by 20 an endogenous protein importin, and promotes the transport of PNA-NLS conjugates into the nucleus. ${ }^{20-22}$ Accordingly, as shown in Supplementary Fig. 6, the PNA1-NLS conjugate labeled with Quasar 570 was shown to be able to localize in the nuclei of human cells. Thus the presence of the Quasar 570 25 fluorophore does not modify the recognition properties of the PNA-peptide conjugate. This localization in nuclei should mean the easy interaction of PNA-NLS conjugate with its target DNA (i.e. genomic DNA) to a certain extent and it might be helpful for in-cell and in vivo applications of PNA-NLS conjugate. This 30 conjugate can be also a starting point to develop the therapeutic transcriptional inhibitors by the antigene strategy.
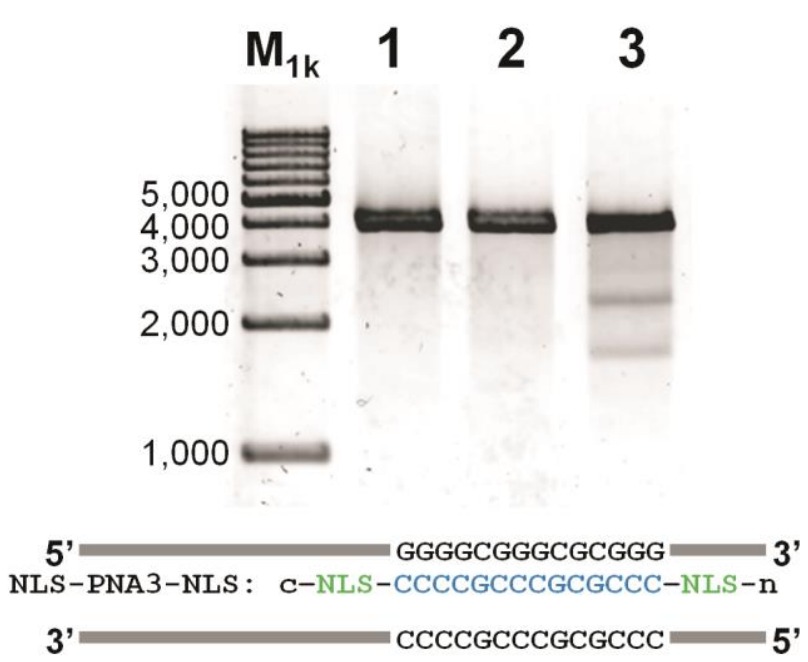

Fig. 5 Site-selective scission of a consecutive GC sequence in $b c l$-2 gene. Lane 1, DNA only; lane 2, Ce(IV)-EDTA treatment; lane 3, the 35 combination of Ce(IV)-EDTA and NLS-PNA3-NLS. Reaction conditions: $[\mathrm{DNA}]=4.0 \mathrm{nM},[\mathrm{NLS}-\mathrm{PNA} 3-\mathrm{NLS}]=200 \mathrm{nM},[\mathrm{Ce}(\mathrm{IV})-$ EDTA $]=200 \mu \mathrm{M},[\operatorname{HEPES}(\mathrm{pH} 7.0)]=5.0 \mathrm{mM}$ and $[\mathrm{NaCl}]=100 \mathrm{mM}$ at $37^{\circ} \mathrm{C}$ for 3 days. The scission mixture was treated with Pvu II before the electrophoresis. The scission site of Pvu II is presented in Supplementary 40 Figure 3.

Site-selective scission of highly GC-rich sequence (bcl-2 gene) by NLS-modified PNA/Ce(IV)-EDTA
In the previously-developed DNA cutter using two PNAs, modified nucleobases (pseudo-complementary bases) are 45 absolutely necessary to preclude the self-hybridization of PNAs and promote its invasion process. This necessity of pcPNAs might make this technology less accessible to scientists who are not familiar with artificial DNAs. By using PNA-NLS conjugate, the present DNA cutter requires no pseudo-complementary PNA 50 and the procedures to apply DNA cutters were dramatically simplified. On the other hand, from the viewpoints of practical applications, another one of the most significant advantages of the present DNA-cutting system is that even highly GC-rich sequences can be successfully targeted. As an example, a 55 consecutive 14-bp GC sequence (5'-GGGGCGGGCGCGGG3'/3'-CCCCGCCCGCGCCC-5'), which is found in a promoter of cancer-related $b c l-2$ gene, was chosen as a target in Fig. 5. An NLS of SV 40 was attached to both the C- and the N-termini of PNA3. As shown in lane 3, the NLS-PNA3-NLS/Ce(IV)-EDTA 60 system clearly accomplished the selective scission at the PNA3 binding-site. The PNA3 conjugate having an NLS only at Cterminus was also effective for the site-selective scission, although the scission was slightly less efficient (Supplementary Figure 5). In spite of the enormous thermodynamic stability of ${ }_{65}$ this targeted sequence, the PNA3 portion in the conjugate successfully binds to the complementary strand through the cooperation with the NLS moiety. It is noteworthy that these extremely GC-rich regions cannot be targeted by the DNA cutter using double-duplex invasion of pcPNA strands, because of 70 unavailability of appropriate pseudo-complementary bases for $\mathrm{G}$ and $\mathrm{C}$ (vide ante). For the present DNA-cutting system, this factor is never an obstacle since it involves only one strand of PNA-NLS conjugate.

\section{Conclusions}

75 It has been shown that the PNA-NLS conjugate is the first singlemolecular activator with conventional PNA to activate target site in double-stranded DNA and promotes selective scission by $\mathrm{Ce}(\mathrm{IV})-\mathrm{EDTA}$ at the site. There is no specific limitation to target sequences and even highly GC-rich regions can be hydrolyzed 80 selectively. The scission site can be straightforwardly determined in terms of the Watson-Crick rule, and no pseudo-complementary nucleobase is necessary. Furthermore, the difference of single base-pair in DNA substrates is clearly distinguished and only the targeted sequence is hydrolyzed. In addition to this, the present 85 results provide a further piece of evidence of the occurrence of duplex invasion by PNA-NLS conjugates into dsDNA, which have been proposed to be the mechanism for transcription inhibition by antigene strategy in cells and in vivo. The NLS peptide not only allows PNA to localize into the nuclei 90 (Supplementary Figure 6), but also, according to our DNAcleavage results, has a decisive role in the sequence selective invasion process. It should be also noted that the reduced number of components in the current DNA cutter system should work better for its preparation and delivery. The versatility and easy

95 availability of the present cutter, as well as its simplicity, are certainly advantages both for previously-developed and various applications in new fields.

\section{Experimental section}




\section{Materials}

The PNA or PNA-NLS conjugates were synthesized using standard Fmoc- or Boc-chemistry-based solid phase peptide synthesis (see reference 24 for PNA1 and PNA1-NLS, and 5 reference 11 for protocols used for the other PNAs). Appropriately-protected PNA monomers were purchased from ASM Research Chemicals and Panagene. These PNAs were purified by reversed-phase HPLC and characterized by MALDI TOF-MS (Bruker, AutoFLEX).

10 The target DNA for the site-selective scission was prepared by conventional methods using overlapping PCR and TOPO ${ }^{\circledR}$ cloning vector (from Life Technologies). After cloning, these plasmid DNAs were purified by QIAprep Spin Miniprep Kit (from Qiagen) and used for the following experiments. The

${ }_{15} \mathrm{Ce}$ (IV)-EDTA complex solution was prepared as follows. ${ }^{6}$ At first, $20 \mathrm{mM}$ solution of $\mathrm{Ce}\left(\mathrm{NH}_{4}\right)_{2}\left(\mathrm{NO}_{3}\right)_{6}$ (from Nacalai Tesque) in water and $20 \mathrm{mM}$ EDTA-4Na (from TCI) in HEPES (from TCI) buffer were mixed. Then the $\mathrm{pH}$ was adjusted to 7.0 with small amounts of $1 \mathrm{M} \mathrm{NaOH}$ (from Wako) solution.

\section{${ }_{20}$ Site-selective scission of double-stranded DNA by PNA- NLS/Ce(IV)-EDTA}

The target double-stranded DNA was first mixed with the PNANLS conjugate at $\mathrm{pH} 7.0$ (HEPES buffer) and these mixtures were incubated at $50^{\circ} \mathrm{C}$ for $1 \mathrm{~h}$. Then, the DNA hydrolysis 25 reaction was started by adding $\mathrm{Ce}$ (IV)/EDTA and $\mathrm{NaCl}$ to final concentrations of $200 \mu \mathrm{M}$ and $100 \mathrm{mM}$, respectively. Typical cleavage conditions were as follows: $[\mathrm{DNA}]=4 \mathrm{nM}$, [PNA or PNA-NLS conjugate $]=200 \mathrm{nM},[$ HEPES $(\mathrm{pH} 7.0)]=5 \mathrm{mM}$, $[\mathrm{NaCl}]=100 \mathrm{mM}$ and $[\mathrm{Ce}(\mathrm{IV})-\mathrm{EDTA}]=200 \mu \mathrm{M}$. After a 30 predetermined time, the reaction was quenched by adding the solution of ethylenediamine- $N, N, N^{\prime}, N^{\prime}$ tetrakis(methylenephosphonic acid) and the mixture was further incubated at $50^{\circ} \mathrm{C}$ for $1 \mathrm{~h}$ to complete the quenching. To identify the sites of the scissions by PNA-NLS/Ce(IV)-EDTA, the 35 scission products were further treated with appropriate restriction enzymes to provide dual scission fragments for the confirmation of scission sites. The scission fragments were evaluated by $0.8 \%$ agarose gel electrophoresis (the sites of the scissions by PNANLS/Ce(IV)-EDTA and the restriction enzymes are presented in ${ }_{40}$ Supplementary Figure 3) and the bands were stained by GelStar ${ }^{\circledR}$ (from FMC).

Fluorescence spectroscopy for the confirmation of complex formation between PNA-NLS conjugate and the target DNA

A Quasar 570 dye (from BIOSEARCH TECHNOLOGIES, INC) 45 was directly conjugated to the terminal amino group of PNA2 backbone through amide linkage (In order to reduce the background fluorescence, a phosphate buffer ( $\mathrm{pH}$ 7.0) was used instead of a HEPES (pH 7.0) buffer; [Dye-PNA2-NLS] $=500 \mathrm{nM}$, [target DNA] $=0$ or $1000 \mathrm{nM}$ and [phosphate buffer $(\mathrm{pH} 7.0)]=5$ $50 \mathrm{mM}$ ). The fluorescence spectra and lifetimes were measured by a photon counting method with a streak scope (Hamamatsu Photonics, C4334-01) using the second harmonic generation (SHG, $395 \mathrm{~nm}$ ) of a Ti:sapphire laser (Spectra-Physics, Tsunami $3950-\mathrm{L} 2 \mathrm{~S}$, fwhm $=150 \mathrm{fs}$ ) as an excitation source.

\section{${ }_{55}$ Acknowledgements}

This work was partially supported by Grants-in-Aid for Specially Promoted Research from the Ministry of Education, Culture, Sports, Science and Technology, Japan (18001001 and 22000007), by the Global COE Program for Chemistry 60 Innovation, and by a Grant from Italian Ministry of Education and Research (MIUR-PRIN09).

\section{Notes and references}

${ }^{a}$ Life Science Center of Tsukuba Advanced Research Alliance, University of Tsukuba, 1-1-1 Tennoudai, Tsukuba, Ibaraki, 305-8577, Japan. Fax: 65 (+)81-29-853-6045; Tel: (+)81-29-853-6045; E-mail:

komiyama@tara.tsukuba.ac.jp

${ }^{b}$ Research Center for Advanced Science and Technology, The University of Tokyo, 4-6-1 Komaba, Meguro-ku, Tokyo, 153-8904, Japan.

${ }^{c}$ Institute of Multidisciplinary Research for Advanced Materials, Tohoku 70 University, Katahira 2-1-1, Aoba-ku, Sendai 980-8577, Japan.

${ }^{d}$ Department of Organic and Industrial Chemistry, University of Parma, Parma, 43124, Italy. Fax: (+)39-0521-905472; Tel: (+)39-0521-905406; E-mail: roberto.corradini@unipr.it

$\dagger$ Electronic Supplementary Information (ESI) available: [Mass analysis 75 of PNA-NLS, details of site-selective DNA scission by PNA-NLS, cleavage maps of the artificial DNA cutter and corresponding restriction enzymes, DNA scission experiment of PNA without NLS, behavior of Dye-PNA-NLS in human cells and fluorescence parameters]. See DOI: $10.1039 / \mathrm{b} 000000 \mathrm{x} /$

$80 \ddagger$ These restriction enzymes were chosen to provide dual scission fragments of appropriate sizes for the gel electrophoresis analysis.

1. T. A. Shell and D. L. Mohler, Curr. Org. Chem., 2007, 11, 1525 1542.

85 2. J. Sumaoka, Y. Yamamoto, Y. Kitamura and M. Komiyama, Curr. Org. Chem., 2007, 11, 463-475.

3. C. L. Liu and L. Wang, Dalton Transactions, 2009, 227-239.

4. F. Mancin, P. Scrimin and P. Tecilla, Chem Commun., 2012, 48, 5545-5559.

90 5. Y. Aiba, J. Sumaoka and M. Komiyama, Chem. Soc. Rev., 2011, 40, 5657-5668.

6. M. Komiyama, Y. Aiba, Y. Yamamoto and J. Sumaoka, Nat. Protoc., 2008, 3, 655-662.

7. Y. Kitamura and M. Komiyama, Nucleic Acids Res., 2002, 30, e102.

95 8. M. Egholm, O. Buchardt, L. Christensen, C. Behrens, S. M. Freier, D. A. Driver, R. H. Berg, S. K. Kim, B. Norden and P. E. Nielsen, Nature, 1993, 365, 566-568.

9. G. Haaima, H. F. Hansen, L. Christensen, O. Dahl and P. E. Nielsen, Nucleic Acids Res., 1997, 25, 4639-4643.

100 10. J. Lohse, O. Dahl and P. E. Nielsen, Proc. Natl. Acad. Sci. U.S.A., 1999, 96, 11804-11808.

11. M. Komiyama, Y. Aiba, T. Ishizuka and J. Sumaoka, Nat. Protoc., 2008, 3, 646-654.

12. K. Ito, H. Katada, N. Shigi and M. Komiyama, Chem. Commun., $105 \quad 2009,6542-6544$.

13. T. Ishizuka, J. Yang, M. Komiyama and Y. Xu, Angew. Chem. Int. Ed., 2012, 51, 7198-7202.

14. G. Lahoud, V. Timoshchuk, A. Lebedev, K. Arar, Y. M. Hou and H. Gamper, Nucleic Acids Res., 2008, 36, 6999-7008.

110 15. S. Hoshika, F. Chen, N. A. Leal and S. A. Benner, Angew. Chem. Int. Ed., 2010, 49, 5554-5557.

16. A. G. Olsen, O. Dahl, A. B. Petersen, J. Nielsen and P. E. Nielsen, Artificial DNA: PNA \& XNA, 2011, 2, 32-36. 
17. X. Zhang, T. Ishihara and D. R. Corey, Nucleic Acids Res., 2000, 28, 3332-3338.

18. V. Chenna, S. Rapireddy, B. Sahu, C. Ausin, E. Pedroso and D. H. Ly, Chembiochem, 2008, 9, 2388-2391.

5 19. G. He, S. Rapireddy, R. Bahal, B. Sahu and D. H. Ly, J. Am. Chem. Soc., 2009, 131, 12088-12090.

20. L. J. Branden, A. J. Mohamed and C. I. E. Smith, Nat. Biotechnol., 1999, 17, 784-787.

21. G. Cutrona, E. M. Carpaneto, M. Ulivi, S. Roncella, O. Landt, M.

10 Ferrarini and L. C. Boffa, Nat. Biotechnol., 2000, 18, 300-303.

22. S. Cogoi, A. Codognotto, V. Rapozzi, N. Meeuwenoord, G. van der Marel and L. E. Xodo, Biochemistry, 2005, 44, 10510-10519.

23. R. Tonelli, S. Purgato, C. Camerin, R. Fronza, F. Bologna, S. Alboresi, M. Franzoni, R. Corradini, S. Sforza, A. Faccini, J. M.

15 Shohet, R. Marchelli and A. Pession, Mol. Cancer Ther., 2005, 4, 779-786.

24. R. Tonelli, A. McIntyre, C. Camerin, Z. S. Walters, K. Di Leo, J. Selfe, S. Purgato, E. Missiaglia, A. Tortori, J. Renshaw, A. Astolfi, K. R. Taylor, S. Serravalle, R. Bishop, C. Nanni, L. J. Valentijn, A.

20 Faccini, I. Leuschners, S. Formica, J. S. Reis-Filho, V. Ambrosini, K. Thway, M. Franzoni, B. Summersgill, R. Marchelli, P. Hrelia, G. Cantelli-Forti, S. Fanti, R. Corradini, A. Pession and J. Shipley, Clin. Cancer Res., 2012, 18, 796-807.

25. A. Faccini, A. Tortori, T. Tedeschi, S. Sforza, R. Tonelli, A. Pession,

25 R. Corradini and R. Marchelli, Chirality, 2008, 20, 494-500.

26. A. Krzyzaniak, M. Siatecka, A. Szyk, P. Mucha, P. Rekowski, G. Kupryszewski and J. Barciszewski, Mol. Biol. Rep., 2000, 27, 51-54.

27. B. J. Harvey and M. Levitus, J. Fluoresc., 2009, 19, 443-448.

\section{Teble of contents}

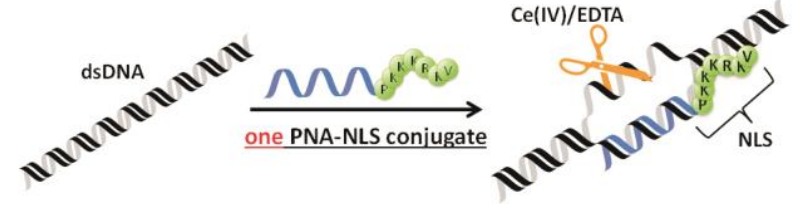

Only one strand of PNA-NLS (Nuclear Localization Signal) 35 conjugate successfully activated the target site in double-stranded DNA and promoted the site-selective scission there by Ce(IV)EDTA. Conventional PNA without pseudo-complementary bases can be utilized for the conjugate. This simple DNA cutter can taget even highly G-C rich sequences and thus is promising for 40 various applications. 